\title{
S'engager « corps et âme »
}

Expériences et carrières militantes des manifestants israéliens contre la barrière de séparation

Committing oneself "heart and soul." Experiences and activist careers of Israeli opponents to the separation fence

\section{Karine Lamarche}

\section{CpenEdition}

Journals

Édition électronique

URL : http://journals.openedition.org/conflits/18120

DOI : $10.4000 /$ conflits. 18120

ISSN : $1777-5345$

Éditeur :

CCLS - Centre d'études sur les conflits lilberté et sécurité, L'Harmattan

Édition imprimée

Date de publication : 5 septembre 2011

Pagination : 125-150

ISBN : 978-2-296-56086-4

ISSN : 1157-996X

\section{Référence électronique}

Karine Lamarche, « S'engager « corps et âme » », Cultures \& Conflits [En ligne], 81-82 | Printemps/Été

2011, mis en ligne le 05 septembre 2012, consulté le 30 mars 2021. URL : http://

journals.openedition.org/conflits/18120; DOI : https://doi.org/10.4000/conflits.18120 


\section{S'engager " corps et âme»}

\section{Expériences et carrières militantes des manifestants israéliens contre la barrière de séparation}

\section{Karine LAMARCHE}

Karine Lamarche est doctorante en sciences sociales (ENS-EHESS, Paris), membre du Centre Maurice Halbwachs et du CeRIES. Elle prépare une thèse sous la direction de Michel Offerlé portant sur l'engagement d'Israéliens contre l'occupation pendant la seconde Intifada.

T 26 décembre 2003, l'armée israélienne tire à balles réelles contre des miliLtants qui tentent de sectionner la barrière de séparation ${ }^{1}$. Gil Naamati, un Israélien de 22 ans originaire d'un kibboutz du sud du pays et ayant terminé son service militaire quelques mois plus tôt, est grièvement blessé. Les médias font leurs gros titres de cet incident et les responsables militaires sont contraints de justifier publiquement l'usage de munitions létales à l'encontre de ressortissants juifs d'Israël, ainsi que l'absence de sommations préalables. Le chef d'état-major tente ainsi d'expliquer l'attitude des soldats en déclarant : « Ils [les militants israéliens] se sont fait passer pour des Arabes, se sont mêlés à des Palestiniens et ont pénétré [...] illégalement du côté palestinien de la barrière 2 ». De leur côté, les militants répondent qu'ils n'ont cessé d'interpeller les soldats en hébreu et que ces derniers ne pouvaient donc pas ignorer qu'ils se trouvaient en présence d'Israéliens ${ }^{3}$. La controverse provoquée par

1. L'année précédente, le gouvernement israélien avait lancé la construction de cette barrière qualifiée aussi de " barrière de sécurité » (gader bitachon). Cf. la page internet du Ministère de la défense israélien consacrée à la dite barrière (URL consulté le 03/12/10) : http://www.securityfence.mod.gov.il/Pages/ENG/operational.htm. Les Palestiniens et les autres détracteurs de la barrière la nomment souvent, de leur côté, " mur de l'apartheid » ou "mur de la honte». Cf. la page internet de la campagne palestinienne « contre le mur de l'apartheid » (URL consulté le 03/12/10) : http://www.stopthewall.org/. Bien que la Cour Internationale de Justice ait décidé de nommer « mur » l'ouvrage construit par Israël, je parlerai pour ma part de "barrière » dans la mesure où, dans les zones concernées par mes observations, la structure était composée d'un grillage et non d'un mur de béton.

2. Déclaration du général Moshe Yaalon, chef d'état-major, selon une source Reuters et AFP rapportée dans : "Des tirs de Tsabal sur un Israélien ont lancé un vif débat en Israël », $L e$ Monde, 29 décembre 2003.

3. Ce « détail » est d'ailleurs confirmé par les images filmées au cours de l'événement comme le montre le documentaire autoproduit par It's all lies : « Democracy isn't built on demonstra- 
cette question de savoir si oui ou non les soldats avaient conscience de tirer sur des compatriotes montre à quel point l'appartenance nationale de ceux qui protestent est déterminante.

L'autre question qui anime à l'époque les débats consiste à savoir qui sont ces jeunes prêts à s'en prendre à la barrière de séparation sous les yeux des soldats de leur propre pays. Au terme de plusieurs années d'Intifada ayant fait des milliers de victimes palestiniennes mais aussi des centaines de victimes côté israélien, la grande majorité de la population juive est favorable à la séparation physique d'avec les Palestiniens ${ }^{4}$. Même au sein de la gauche, les critiques qui se font entendre portent le plus souvent sur le tracé de la barrière ${ }^{5}$, non sur son principe en lui-même. Il faut dire que beaucoup de ceux qui prônaient jusqu'alors la paix et le dialogue appréhendent la seconde Intifada comme le signe que les Palestiniens n'ont, en fait, jamais voulu la paix. Le mouvement «La Paix Maintenant» (Shalom Achshav 6), qui était parvenu à drainer dans ses rangs des dizaines de milliers de militants dans les années 1980 et 1990 reste silencieux, se refusant à critiquer ouvertement l'armée et le gouvernement. Ce sont principalement de nouveaux mouvements qui vont permettre à ceux n'adhérant pas au discours ambiant sur la «trahison palestinienne » d'exprimer, dans l'action, leur désaccord avec la politique israélienne et la répression militaire dans les territoires occupés - à l'exception de certains anciens groupes comme l'AIC (Alternative Information Center), les « Femmes en Noir » (Nashim beShachor), ou encore «Le Bloc de la Paix » (Gush Shalom).

Pendant la tempête médiatique provoquée par la blessure de Gil Naamati, les Israéliens qui se joignent aux manifestations palestiniennes contre la barrière sont désignés par les médias sous le nom d' "Anarchistes contre le Mur ${ }^{7}$ ». Durant les années suivantes, c'est à eux que la plupart des activités menées dans les territoires palestiniens impliquant des Juifs d'Israël seront imputées. Les manifestations qui ont lieu chaque semaine depuis plu-

tors' bodies » (32 minutes). URL : http://www.archive.org/details/the_right_to_protest

4. Parizot C., "Après le mur : les représentations israéliennes de la séparation avec les Palestiniens », Cultures \& Conflits, 73, Printemps 2009, pp 57-58.

5. Sur les quelques $700 \mathrm{~km}$ de barrière/mur prévus, une partie s'éloigne de la ligne verte (ligne d'armistice de 1949 entre Israël et la Transjordanie) pour s'enfoncer dans le territoire palestinien. Pour une carte de la barrière, voir le site de B'tselem, une ONG israélienne de défense des droits de l'homme (URL consulté le 03/12/10) : http://www.btselem.org/Download/Separation_Barrier_Map_Eng.pdf

6. Les lettres hébraïques «kaf » et " het », qui se prononcent normalement de façon gutturale et fricative (comme la jota espagnole), ont été transcrites ici par le son «ch » (à différencier du son «sh»).

7. D’autres appellations avaient également été utilisées comme les «Juifs contre les Ghettos » ou le "Groupe de Mash'a », mais l'attention médiatique qui a entouré les activités de ces militants au moment de l'affaire Gil Naamati contribue à expliquer qu'ils aient décidé de garder ce nom. Voir Lakoff A., "Israeli Anarchism - Being Young, Queer, and Radical in the Promised Land: an Interview with Yossi”, Anarchist Studies, 13, 2, 2005 (consulté en ligne : http://aaron.resist.ca/?q=node/33) ; Snitz K., "On Recent Palestinian Resistance and its Israeli Support", Brochure sur les "Anarchistes contre le Mur », 2008. Document personnel de l'auteur. 
sieurs années dans le petit village de Bil'in, devenu un symbole de la lutte conjointe, ainsi que dans d'autres villages de Cisjordanie sont connues pour être le point de ralliement de ces « anarchistes », qui participent également à des actions directes non-violentes : blocages de routes, démontages des roadblocks ${ }^{8}$, sabotages de la barrière, constructions d'avant-postes palestiniens, etc. Pourtant, si certaines figures centrales du groupe sont engagées depuis plusieurs années dans des collectifs punk, queer ${ }^{9}$, squatter, et/ou anti-spéciste ${ }^{10}$, dont la sensibilité anarchiste est clairement affichée, les Israéliens participant aux activités des "Anarchistes contre le Mur » sont loin de tous se revendiquer de cette mouvance politique. Le groupe, resté informel 11 depuis sa naissance médiatique, n'exige pas de fidélité à un programme idéologique particulier et n'importe qui peut le rejoindre, ponctuellement ou régulièrement, dès lors qu'il s'oppose à l'occupation et au mur ${ }^{12}$.

La participation à ces protestations est l'une des formes de militantisme les plus risquées et coûteuses qu'un militant contre l'occupation puisse choisir aujourd'hui. En effet, on verra qu'il s'agit d'un engagement chronophage (entre une demi-journée de temps en temps et plusieurs heures par jour pour les plus engagés), financièrement coûteux (participation aux trajets, frais de téléphone portable et surtout frais d'avocats en cas d'arrestation ${ }^{13}$ ), moralement et physiquement épuisant, mais comportant aussi un degré potentiellement élevé de risques d'ordre légal, psychologique, physique, et social. De plus, la participation à ce type d'activités fait l'objet d'une forte condamnation en Israël : les militants anti-occupation sont souvent accusés de mettre en danger la sécurité de leurs concitoyens et d'attiser l'antisémitisme en propageant, à l'extérieur du pays, un discours antipatriotique. La question de savoir ce qui les amène à prendre part à ces protestations sera traitée dans un second temps à travers trois profils de militants ${ }^{14}$ contre la barrière, analysés en termes de

8. Structures de bétons placées par l'armée à l'entrée d'un village et destinées à en bloquer l'accès.

9. Ce mot anglais signifiant « étrange » et utilisé à l'origine comme insulte à l'égard des homosexuels, des transsexuels, des bisexuels, a été récupéré et revendiqué par les opposants à l'hétérocentrisme. Pour une présentation synthétique de la pensée queer, voir Bourcier M-H., Queer Zone, Paris , Balland, 2001.

10. L'anti-spécisme est l'opposition au spécisme, lui-même défini comme suit dans les cahiers anti-spécistes : «Le spécisme est l'idéologie qui justifie et impose l'exploitation et l'utilisation des animaux par les humains de manières qui ne seraient pas acceptées si les victimes étaient humaines. » URL (consulté le 10 août 2010) : http://www.cahiersantispecistes.org/spip.php?article13

11. Il ne possède ni porte-parole, ni bureau, ni salarié. Aucun système d'adhésion ne vient sanctionner l'appartenance à celui-ci.

12. S'il fallait donner une estimation du nombre de militants qui gravitent autour de ce groupe, on pourrait dire qu'il varie, en fonction du degré d'implication, entre quelques dizaines et quelques centaines de participants plus ou moins réguliers. Ce chiffre, volontairement très approximatif, ne tient pas compte des sympathisants ni de ceux qui, ayant été actifs à un moment donné dans les activités du groupe, se sont désengagés (selon les observations menées lors d'une vingtaine d'actions des « Anarchistes contre le Mur », ainsi que pendant plusieurs réunions à Tel-Aviv).

13. Les frais d'avocats sont généralement pris en charge par le groupe des « Anarchistes contre le Mur », grâce à l'argent récolté lors de tournées de soutien en Europe et aux États-Unis. 
«carrières ». Penser l'engagement dont il est question ici à l'aide de ce concept qui renvoie à l'héritage interactionniste ${ }^{15}$, c'est proposer une lecture des continuités et discontinuités, moments de rupture et bifurcations biographiques qui ont rendu possible la participation régulière d'Israéliens aux manifestations contre la barrière de séparation.

Cet article repose sur la constitution d'un corpus empirique constitué des éléments suivants : un carnet de terrain relatant une série d'observations effectuée entre 2006 et 2010 en Isrä̈l/Palestine, ainsi qu'une vingtaine d'entretiens biographiques avec des militants israéliens contre la barrière ${ }^{16}$.

\section{Penser sociologiquement l'engagement israélien contre la barrière}

\section{Risques et cô̂ts d'une "guerre symbolique »}

Chaque semaine depuis cinq ans, après la prière du vendredi, quelques centaines de manifestants sortent du petit village de Bil'in ${ }^{17}$ et se dirigent vers la barrière de séparation. Certains portent le keffieh et arborent le drapeau palestinien, d'autres des pancartes en arabe, anglais ou hébreu. Plusieurs journalistes, photographes et vidéastes sont généralement présents sur place, parfois équipés de masques à gaz et de casques, ce qui donne à la procession une allure guerrière. Sur la route qui quitte le village en serpentant entre les oliviers pour arriver jusqu'à la barrière, les manifestants sont parfois ralentis par les gaz lacrymogènes tirés par l'armée. A d'autres moments, l'intervention des soldats ne survient qu'après avoir laissé la protestation suivre son cours pendant un certain temps, puis décrété la zone "militairement fermée 18 ». En fonction des périodes, de l'unité militaire en place, du climat politique, le déroulement des manifestations diffère grandement. Il est néanmoins rare que la répression ne fasse aucun blessé ${ }^{19}$.

14. Les trois militants en question sont des hommes, choisis en raison de l'intérêt typologique de leurs carrières. Pour autant, les femmes sont présentes dans les manifestations contre la barrière et leur présence n'est pas sans soulever un certain nombre de questions dans la mise en place concrète de la lutte conjointe qu'il serait particulièrement pertinent d'analyser. Cependant, ce point ne sera pas traité dans le cadre de cet article.

15. Initialement employé par Hughes à propos des professions (Hughes E., Men and their Work, Glencoe, The Free Press, 1958), le concept de " carrière » a été repris par Becker dans ses travaux sur la déviance (Becker H., Outsiders. Etudes de sociologie de la déviance, Paris, Métailié, 1985 [1 ère édition en anglais : 1963]). Plus récemment, il a été mobilisé par des sociologues des mouvements sociaux pour analyser l'engagement militant (voir notamment le numéro spécial : «Devenir militants », Revue Française de Science Politique, 51, 1-2, 2001).

16. Sur la soixantaine que compte mon enquête de thèse qui porte, plus généralement, sur les Israéliens engagés contre l'occupation.

17. Situé à une dizaine de kilomètres de la ville palestinienne de Ramallah, celui-ci compte environ mille huit cents habitants. Les remarques à venir concernent principalement ce village, même si certaines observations ont pu être menées dans d'autres, notamment à Ne'alin.

18. L'armée a la possibilité d'émettre un mandat dit de «zone militaire fermée » (shetach zva'i sagour), qui lui permet d'interdire l'accès à un espace déterminé (apparaissant sur une carte) de ceux qu'elle y juge indésirables. C'est l'une des principales mesures de dispersion de la foule justifiant, auprès des tribunaux, les arrestations et les tirs en cas de refus des personnes visées d'évacuer les lieux. 
Gaz lacrymogènes, balles en caoutchouc et grenades assourdissantes ainsi que coups de matraque sont les moyens les plus couramment employés pour disperser les manifestations contre la barrière. A certaines occasions, l'armée utilise également des canons projetant eau, sel, et liquide nauséabond ${ }^{20}$, un engin surnommé « le cri 21 » et, exceptionnellement au cours de la manifestation ${ }^{22}$, des munitions létales. Mais ces dernières ne sont pas les seules à tuer. En 2009, Bassam Abu Rahme du village de Bil'in a péri après avoir reçu une grenade lacrymogène en pleine poitrine à une distance non réglementaire. D’autres ont été tués par des balles en caoutchouc tirées au niveau de la tête. À ce jour, aucun Israélien ni volontaire international n'a perdu la vie au cours d'une manifestation contre la barrière ${ }^{23}$. C'est en revanche le cas de seize Palestiniens entre 2004 et 2009 24. L'avocate israélo-américaine Emily Schaeffer, qui travaille dans le cabinet de Michael Sfard spécialisé dans la défense des organisations de défense des droits de l'homme, des villages en lutte contre la barrière et des militants inculpés, explique dans un entretien :

«La présence des Israéliens change les ordres militaires que l'armée israélienne donne en termes de réponse aux manifestants. Cela signifie que [les soldats] n'ont pas le droit de tirer des munitions létales et qu'ils sont censés éviter autant que possible de tirer même des balles en caoutchouc, ce qui a pour effet de rendre les manifestations bien plus sûres pour toutes les personnes impliquées. 25 ”

Mais s'ils risquent moins leur vie que les Palestiniens, les Israéliens participant aux protestations contre la barrière font néanmoins face à des dangers dont certains peuvent avoir un impact durable sur leur vie. Nombre d'entre

19. Bil'in et Ne'alin sont les deux villages dans lesquels les confrontations entre armée et manifestants sont généralement les plus violentes. À Ma’asara, autre village en lutte contre la barrière, les manifestations se déroulent en général plus calmement.

20. Celui-ci est désigné par le terme "putois » (bo’ech en hébreu) car il reproduit synthétiquement l'odeur émise par l'animal. Son pouvoir odorant est si fort qu'il resterait sur les habits des manifestants jusqu'à cinq ans après les avoir atteints.

21. Tzeaka en hébreu : son assourdissant diffusé pendant une minute par un appareil placé sur un véhicule de l'armée qui provoque des nausées et des pertes d'équilibre.

22. L'armée utilise souvent ce type de munitions lorsqu'elle est sûre de n'avoir à faire qu'à des Palestiniens, typiquement à la fin des manifestations, lorsque la foule s'est dispersée et que seuls restent les jeunes du village, ainsi que lors d'opérations de représailles.

23. Plusieurs ont néanmoins été grièvement blessés. Voir Traubmann T., "Fence protester hit in the head by tear gas canister", Ha'aretz, 5 avril 2005 ; Peled A., "Outside the Fence", Yediot Aharonot, 14 avril 2006 ; Beit-Or-Meital Y., "Injured anti-fence 'anarchist' speaks out", Ynet, 25 février 2006 ; Karpel D., "One blow to the brain”, Ha'aretz, 14 septembre 2006.

24. Voir "Repression allowed. Resistance denied. Israel's suppression of the popular movement against the Apartheid Annexation Wall”, rapport mis en ligne par Addameer (Association de soutien aux prisonniers palestiniens) et le Palestinian Grassroots Anti-Apartheid Wall Campaign, 9 juillet 2009, p. 27. URL (consulté le 9 septembre 2010) : http://www.stopthewall.org/downloads/pdf/repress.pdf

25. Ramsey J., "Canadian companies face legal action for settlement building in Palestine", Rabble, 22 juin 2009, URL (consulté le 8 novembre 2010) : http://rabble.ca/news/2009/06/canadian-companies-face-legal-action-settlement-buildingpalestine 
eux ont été blessés, parfois grièvement. Certains souffrent de problèmes respiratoires en raison de l'inhalation de gaz, d'autres de troubles psychologiques 26 liés à l'exposition répétée à ces situations de violence. Les arrestations sont monnaie courante bien que, en leur qualité de citoyens d'Israël, ils ne passent généralement que quelques heures au poste de police, à la différence des Palestiniens ${ }^{27}$. Les chefs d'accusation les plus fréquents sont « rassemblement illégal », « agression » ou encore « rébellion » et ils risquent généralement une amende ou une caution de 1000 à 10000 shekels (de 200 à 2000 euros environ), qui leur sera rendue sous réserve de ne plus pénétrer dans la zone où ils ont été arrêtés pendant une durée définie par le juge - généralement quelques semaines 28. L'ouverture d'un dossier judiciaire ou l'inscription sur liste noire ${ }^{29}$ peut néanmoins avoir des conséquences lourdes sur la poursuite de leurs études ou sur leur avenir professionnel.

Malgré la violence de ces situations, certains habitués de Bil'in ou de Ne'alin s'y soumettent comme si cela faisait « partie du jeu ». Alors que les gaz lacrymogènes projetés dès le début des manifestations suffisent généralement à décourager les moins aguerris, ceux-là continuent leur chemin vers les soldats, bravant les nuages de fumée et les pierres lancées par les jeunes du village qui commencent à voler autour d'eux. Ils reviennent parfois avec une balle en caoutchouc logée dans la hanche ; le plus souvent avec quelques égratignures. Ils font ainsi la preuve du « dépassement de soi » dont ils sont capables auprès des Palestiniens, qui les valorisent en retour pour leur courage et leur volonté de s'exposer aux mêmes risques qu'eux. Chez ces « guerriers 30 » de la protestation, la peur et le traumatisme ressentis par beaucoup de manifestants moins entraînés sont souvent relégués au second plan, voire dénigrés, comme s’il était honteux de ne pouvoir supporter pendant quelques heures une oppression que les Palestiniens endurent quotidiennement ${ }^{31}$. Le groupe a néanmoins fait, ces dernières années, des efforts pour prendre en compte la souffrance engendrée par ces confrontations violentes, en mettant par exemple en place une équipe de gestion du stress post-traumatique ou en proposant aux nouveaux venus de se faire accompagner par une personne expérimentée. Pour autant, cette forme de militantisme implique d'accepter, au moins théoriquement, de se mettre en danger physiquement mais aussi légalement, psychologiquement ou encore socialement au nom d'une lutte qui se revendique pourtant comme non-violente.

26. Voir Gordon U., "Israeli Anarchism. Statist dilemmas and the dynamics of joint struggle", Anarchist Studies, 15, 1, 2007, p. 24.

27. Les Israéliens dépendent de la juridiction civile. Les Palestiniens, eux, sont soumis à la juridiction militaire et peuvent donc être gardés plusieurs jours avant d'être présentés à un juge.

28. "Repression allowed", op. cit.

29. Reshima Shchoura, en hébreu. Liste établie par les services secrets intérieurs israéliens, le Shin Bet (ou shabak). Voir Blau U., "IDF asks police, Shin Bet for info on left-wing figures active in W. Bank", Ha'aretz, 7 novembre 2008.

30. Cette expression est utilisée notamment par certains militants anti-occupation qui ne participent pas aux manifestations contre la barrière.

31. Voir Gordon U., op. cit., p. 24. 
L'utilisation du label «non-violent » pour qualifier les manifestations contre la barrière semble être le fruit d'une importation de vocabulaire protestataire international dans la mesure où la culture de la résistance palestinienne privilégie la distinction entre lutte armée et lutte populaire à celle opérée entre violence et non-violence. Toute la question est donc d'évaluer la pertinence des mots « violence» et «non-violence» dans le cas qui nous intéresse. Si la définition du premier se heurte à des enjeux socialement construits, il en va de même du second. Gene Sharp, à travers la notion de "Jiu-jitsu politique », a développé l'idée que ceux qui ont recours à des formes d'action non-violentes se servent en fait de la force de leur adversaire pour le déséquilibrer, en le contraignant à recourir à des moyens illégitimes de coercition qui lui échappent ${ }^{32}$. Les participants aux manifestations contre la barrière mettent généralement un point d'honneur à qualifier ces dernières de non-violentes, bien qu'elles soient quasi-systématiquement le thêâtre de débordements sanglants de la part des forces de l'ordre qui ouvrent le feu sans retenue et de la part des jeunes du village qui lancent en retour des pierres dans leur direction.

Alors que de nombreux travaux publiés ces dernières années ont décrit des actions collectives de plus en plus souvent traversées, dans les démocraties occidentales, par un processus de pacification du jeu politique, par une euphémisation et un contrôle élevé de la violence, dont la forclusion passe notamment par l'acte électoral ${ }^{33}$, bref par une " civilisation des mœurs protestataires ${ }^{34}$ », les manifestations contre la barrière semblent ainsi fournir un exemple intéressant de dépacification du jeu politique. Certes, le contexte et le lieu dans lesquels elles se déroulent - la Cisjordanie, occupée depuis une quarantaine d'années par Israël et soumise à la juridiction militaire - ne peuvent pas permettre d'analyser ces manifestations avec les outils mobilisés en sociologie des mobilisations pour analyser les contextes européens et nord-américains marqués par une certaine tolérance à l'égard de la contestation. Mais si la contrainte est fortement présente, ces manifestations ne sont pas comparables non plus à celles qui peuvent s'observer dans des situations caractérisées par l'autoritarisme du régime en place ${ }^{35}$, notamment du fait de la présence d'Israéliens et d'internationaux en leur sein.

32. Sharp G., The Politics of Non Violent Action, Boston, Extending Horizons Books, 1973.

33. Ihl O., "La civilité électorale : vote et forclusion de la violence en France », Cultures $\varepsilon$ Conflits, 9-10, 1993, pp. 75-87.

34. Memmi D., "Le corps protestataire aujourd'hui : une économie de la menace et de la présence », Sociétés contemporaines, 31, 1998, p. 87.

35. Pour une réflexion sur l'engagement en contexte autoritaire (et une réflexion sur les limites du concept d'autoritarisme), voir le dossier : "S'engager en situation de contrainte », Genèses, 4, 77, 2009. 
D’ailleurs, le fait que la Cour Suprême israélienne (Bagatz) ait déclaré illégales certaines parties du tracé de la barrière et demandé leur modification comme à Beit Surik, Budrus et Bil'in, montre que les opposants au mur disposent, en Israël même, de recours juridiques pour faire avancer leur cause. Pour autant, manifester reste une ressource importante, notamment en raison du symbole fort que véhicule le rassemblement d'Israéliens et de Palestiniens dans une même lutte et du potentiel de publicisation que celui-ci leur assure auprès du public international. Michel Offerlé a montré que les acteurs sociaux qui se mobilisent peuvent puiser alternativement dans trois types de ressources : le nombre (faire masse), la science (avoir recours à l'expertise) et la vertu (scandaliser) ${ }^{36}$. Si le recours à l'expertise est ponctuellement utilisé par des organisations comme « La Paix Maintenant » ou B'tselem ${ }^{37}$ pour contester le tracé du mur auprès de la Cour Suprême, il est très rare que les militants anti-occupation parviennent à rassembler massivement autour de leur cause. Cela peut contribuer à expliquer le recours à la vertu : scandaliser, en se confrontant aux forces de l'ordre ou en introduisant des registres « tapageurs » et festifs dans le répertoire d'action permet en effet de compenser le faible nombre de participants aux manifestations hebdomadaires contre la barrière de séparation.

Celles-ci constituent en effet un exemple intéressant de «manifestations de papier 38 » dans la mesure où elles visent à attirer l'attention des journalistes pour occuper l'espace médiatique et imposer la mise sur agenda d'un problème public ou politique. Le fait qu'elles se déroulent loin de l'espace habituellement investi par les manifestants, la rue, avec quelques centaines d'oliviers pour seuls spectateurs et que tous les protagonistes présents sur placesoldats, villageois et partenaires de lutte israéliens et internationaux - en soient partie prenante 39 vient encore renforcer l'importance des médias dans la recherche de visibilité. Ceci explique en partie le développement de formes d'action festives, théâtrales, impertinentes, et/ou humoristiques destinées à attirer l'attention des journalistes. En 2010, les habitants du village de Bil'in se sont par exemple déguisés en personnages du film Avatar et les images prises cette semaine-là au cours de la manifestation ont fait le tour du monde.

36. Offerlé M., Sociologie des groupes d'intérêt, Paris, Montchrestien, pp. 110-125, 1998 [11ère éd. : 1994].

37. "A l'image de " en hébreu (tiré de la Genèse). B'tselem, créé en 1989 en pleine première Intifada, se présente comme le centre israélien d'information pour les droits de l'homme dans les territoires occupés.

38. Champagne P., «La manifestation. La production de l'événement politique », Actes de la Recherche en Sciences Sociales, 52-53, 1984, p. 28 et ss.

39. Cette affirmation pourrait toutefois être nuancée par le fait que, dans certains villages, les habitants des colonies proches de la barrière en construction ont parfois l'occasion d'entendre, de voir et même de sentir l'odeur des manifestations. Ainsi, à Hashmonaim, des colons s'étaient plaints de ne pouvoir profiter de leur jardin le vendredi en raison de l'odeur agressive des gaz lacrymogènes tirés sur les manifestants contre la barrière. Voir Kyzer L., "As Na'alin protests fence, Hashmonaim residents hide", Ha'aretz, 19 octobre 2009. 
Depuis le début de la construction de la barrière de séparation, des centaines d'articles ont été publiés dans les journaux du monde entier sur les manifestations conjointes contre la barrière et sur ceux qui y participent. Dans les entretiens consacrés aux Israéliens, l'une des questions récurrentes est : «Qu'est-ce qui vous pousse à participer à ces manifestations ? » Les réponses données sont d'ordre moral, mais aussi stratégique et symbolique. On peut tout d'abord manifester contre la barrière parce qu'on la considère comme injuste et que l'on refuse d'être associé, en tant qu'Israélien, à cette entreprise de séparation. Protester contre la barrière peut aussi renvoyer à la volonté d'avoir un impact direct sur le comportement des soldats : comme on l'a déjà évoqué, la présence d'Israéliens lors des manifestations permet une répression plus pondérée. Elle joue également sur la couverture médiatique : lorsque ce sont des Israéliens qui sont blessés ou arrêtés, la presse est plus encline à parler des protestations. Enfin, certains affirment participer aux manifestations aux côtés des Palestiniens pour leur témoigner une solidarité concrète en se confrontant à leurs côtés à la violence de l'armée ou parce qu'ils ressentent de la culpabilité du fait de leur appartenance au « camp des occupants ». La participation a donc ici plus une visée symbolique, celle d'affirmer une égalité qui n'existe pas dans les faits entre Israéliens et Palestiniens, en récusant l'étiquette nationale comme principal marqueur identitaire et comme garantie d'une vie plus facile.

Bien que la dimension égocentrique soit indirectement soulignée par ceux qui déclarent militer pour « être en paix avec [leur] conscience 40 », les raisons d'agir sont généralement présentées sous l'angle de l'altruisme, du dévouement pour des valeurs nobles, du refus de l'injustice. Or, il semble nécessaire de s'affranchir au maximum des discours enchantés validant moralement la participation aux activités contre la barrière si l'on veut espérer donner un sens sociologique aux trajectoires militantes. «Les univers militants sont officiellement - c'est-à-dire de manière autorisée, légitimement, publiquement et collectivement - "désintéressés” ${ }^{41}$ ", souligne Daniel Gaxie, qui défend l'idée que des rétributions matérielles et symboliques peuvent consciemment et, plus généralement inconsciemment, amener des individus à participer à des actions collectives. Les services rendus par les syndicats ou les associations dans lesquelles on milite, les emplois auxquels ils peuvent permettre d'accéder, etc. sont certes à prendre en considération ${ }^{42}$, mais il faut $\mathrm{y}$ ajouter ce qui relève «des satisfactions, des avantages, des plaisirs, des joies, des bonheurs, des pro-

40. Formule employée par plusieurs manifestants contre la barrière lors de discussions informelles.

41. Gaxie D., «Rétributions du militantisme et paradoxes de l'action collective », Swiss Political Science Review, 11, 1, 2005. p. 8.

42. Mancur Olson est le premier à avoir souligné l'importance de ces « incitations sélectives ». Olson M., La logique de l'action collective, Paris, PUF, 1978 [1 ère édition en anglais : 1965]. 
fits, des bénéfices, des gratifications, des incitations, ou des récompenses du militantisme 43 ». On trouve parmi ceux-ci les sentiments positifs qui accompagnent le fait d'accomplir une action en accord avec ses valeurs, de faire partie d'un groupe dont on partage les principes, de développer de nouvelles amitiés voire accointances amoureuses, de vivre des expériences militantes grisantes, etc. Cette approche a l'avantage de permettre une prise de distance avec les justifications morales avancées par les militants et de proposer une analyse des déterminants de l'engagement individuel. Elle comporte néanmoins le risque d'être tautologique puisque les issues positives de la participation à une action collective, qu'elles aient été ou non recherchées par les acteurs, sont systématiquement analysées a posteriori comme des rétributions permettant d'expliquer cette participation. De plus, il semblerait pertinent d'appréhender les bénéfices tirés du militantisme comme un «facteur de stabilisation de l'engagement » plutôt que comme un «facteur d'engagement » dans la mesure où " les militants, avant de rejoindre une organisation, sont généralement dans une situation d'inconnu et ne perçoivent pas toujours les rétributions que peut leur apporter leur engagement ${ }^{4} »$. Enfin, l'approche en termes de rétributions, pour nécessaire qu'elle soit dans l'analyse de nombreuses situations d'engagement, se heurte à des difficultés analytiques lorsqu'il s'agit de comprendre le militantisme à haut risque, dans lequel les dangers potentiels et les coûts de la participation sont a priori infiniment plus élevés que les éventuels bénéfices.

Doug McAdam a proposé, dans son étude sur les participants à la campagne du Freedom Summer, un modèle permettant d'expliquer le recrutement dans des formes de militantisme risquées et coûteuses ${ }^{45}$. Selon lui, avant de s'engager dans ce type d'activités, les acteurs commencent généralement par le faire là où risques et coûts sont moins élevés, encouragés par deux facteurs facilitateurs : des attitudes politiques réceptives d'une part, généralement acquises au cours des différentes phases de la socialisation, et des contacts avec d'autres militants d'autre part. Cet engagement initial ne conduit pas nécessairement au militantisme à haut risque. Il faut notamment pour cela que les militants soient relativement libres de contraintes personnelles et professionnelles. Ce modèle fonctionne pour la plupart des militants contre la barrière mais l'importance des différents éléments varie beaucoup d'un cas à l'autre. En insistant sur la place occupée par l'engagement dans le réseau plus large de relations sociales entretenues par chacun des trois militants dont les trajectoires sont retracées ci-dessous, il s'agit d'expliquer l'entrée, le maintien puis éventuellement le désengagement de cette forme spécifique de militantisme qu'est la participation aux manifestations contre la barrière de séparation.

43. Gaxie D., op. cit., p. 4.

44. Passy F., L'action altruiste, Genève, Droz, 1998, p. 196.

45. McAdam D., "Recruitment to High Risk Activism: The Case of Freedom Summer", The American Journal of Sociology, 92, 1, 1986, pp. 64-90. 
Précisons qu'il ne s'agit pas ici de montrer qu'il existe un début et une fin à la « carrière de manifestant contre la barrière » entre lesquels se déploierait une série d'événements sur un mode linéaire, ni que les parcours individuels prennent forme indépendamment des institutions qui leur préexistent. L'analyse processuelle de l'engagement vise au contraire à montrer que ce sont souvent des micro-événements, des rencontres, des situations nouvelles, bref un enchainement de facteurs qui, mis bout à bout, permettent à un individu de «franchir le pas », puis de faire l'apprentissage d'un nouveau rôle.

Le premier profil est constitué des « militants multipositionnés 46 ». Ceuxci ont comme caractéristiques d'être fortement politisés, de cumuler des engagements polyvalents dans différentes luttes sociales et politiques et de déjà appartenir, au moment où ils s'engagent, à un milieu militant marqué par des valeurs, des pratiques et un mode de vie alternatifs voire en rupture avec leur socialisation initiale. Il s'agit généralement des militants déjà engagés en 2003 lors des premières manifestations contre la barrière. Le second profil est composé de ceux qu'on pourrait qualifier de «militants pragmatistes ». Leur engagement contre l'occupation est généralement plus ancien que la participation aux manifestations contre la barrière, qu'ils ont rejointes seulement entre 2005 et 2007 , à une époque où manifester contre la barrière était devenu l'une des seules options pour ceux qui souhaitaient exprimer dans l'action leur opposition à l'occupation. Pour la plupart liés au monde universitaire, ils se revendiquent souvent de la gauche non-sioniste mais ne se considèrent pas pour autant anarchistes. Enfin, le dernier type est celui des «militants néophytes » qui sont arrivés dans les manifestations contre la barrière suite à un enchaînement de facteurs que leur socialisation politique familiale aurait difficilement permis d'anticiper. En effet, ces derniers sont les moins politisés des militants contre la barrière. Leurs premiers pas dans les manifestations se font généralement avec beaucoup d'appréhension et les rencontres qu'ils y font tiennent une place déterminante dans la poursuite ou non de leur engagement.

\section{Yaniv, Ido et Doron, trois militants contre la barrière 47}

\section{"Être sur tous les fronts ». L'engagement total d'un militant multipositionné}

En avril 2003, alors que les terres du village palestinien de Mash'a commencent à être confisquées par le gouvernement israélien pour construire la barrière de séparation, un campement est installé pour permettre à ceux qui le souhaitent de venir s'informer et de réfléchir ensemble à la manière de lutter. À partir de l'été suivant, des manifestations sont organisées par les comités populaires des villages concernés et voient converger en leur sein des militants

46. Cette expression a déjà été utilisée dans d'autres travaux sur le militantisme, notamment : Péchu C., "Les générations militantes à Droit Au Logement », Revue Française de Science Politique, 51, 1-2, 2001, p. 76.

47. Pour des raisons d'anonymat, les prénoms ont été modifiés. 
palestiniens, israéliens et des volontaires internationaux principalement affiliés à l'International Solidarity Movement (ISM) 48. Parmi les Israéliens, beaucoup appartiennent au milieu anarchiste de Tel-Aviv et cumulent un mode de vie alternatif (vie en squat ou en collectivité, végétalisme, anti-consumérisme, etc.), des convictions anti-spécistes, anti-autoritaristes et anti-patriarcales, une adhésion à la cause queer et une forte opposition à la politique partisane. Comment dès lors expliquer qu'ils aient décidé de se joindre à la lutte de villageois palestiniens que leurs quotidien et traditions placent à l'opposé de ces schémas idéologiques et de leur application concrète au jour le jour, sans même parler de la contradiction apparente à ce que des anarchistes soutiennent la création d'un État ${ }^{49}$ ? L'analyse de la carrière d'un de ces militants permettra de répondre à cette question tout en précisant les particularités de cette catégorie de «militants multipositionnés».

Yaniv est né en 1976 d'un père étatsunien et d'une mère française ayant tous les deux immigré en Israël. Dès l'âge de dix ans, il commence à s'intéresser aux idées d'extrême-gauche par le biais de son frère aîné, lui-même très engagé politiquement, qui lui fournit un accès à des sources d'information alternatives. À l'adolescence, il découvre lors de vacances d'été le milieu anarchiste parisien, auquel il s'identifie très rapidement au point de ressentir assez vite un fort décalage vis-à-vis de sa propre société :

"C'était le début des années 1990. Il y a des personnes qui m’ont parlé des squats. Je savais même pas ce que ça voulait dire... J'ai commencé à me poser des questions là-dessus, sur l'activisme, sur l'anarchisme, le féminisme, tous ces trucs. Et même sur l'insoumission militaire dont on parlait là-bas... [...] L'énergie que j'avais en moi, souvent c'était en pensant à ces gens-là, ceux qui étaient en France. Quand je revenais en Israël, j'étais très seul. Je me sentais comme quelqu'un qui était en prison. »

Ayant atteint l'âge de la conscription, Yaniv parvient à être exempté pour motifs psychologiques. A l'époque, il n'existe pas encore de mouvement de soutien aux jeunes objecteurs de conscience ${ }^{50}$ et ceux qui ne veulent pas servir dans l'armée n'ont comme seule alternative à l'enrôlement ou à la prison que de simuler ou d'exagérer, devant un psychologue, des problèmes personnels, une faiblesse mentale, etc. Les jeunes qui sont exemptés sur cette base sont classés dans la catégorie «Profil 2151 », qui rend difficile, voire impossible la

48. Organisation palestinienne fondée en 2001 qui rassemble des volontaires internationaux venant principalement d'Europe et des États-Unis pour soutenir les Palestiniens dans leur lutte contre l'occupation.

49. Cette question a été soulevée par Uri Gordon. Voir Gordon U., op. cit., pp. 14-15.

50. À la fin des années 1990, le groupe Profil Chadash (« Nouveau Profil») avait été formé pour promouvoir la démilitarisation de la société israélienne, notamment en apportant son soutien aux objecteurs de conscience. Avant cela, le mouvement Yesh Gvul avait soutenu les Refuzniks (principalement des réservistes) de la guerre du Liban et de la première Intifada. 
poursuite de certaines études ou l'accès à certains emplois. Mais le stigmate 52 peut aussi fonctionner, pour la personne qui en est victime, comme une « base d'organisation pour sa vie 53 » et l'amener à renforcer son identification à une communauté d'appartenance composée de pairs qui lui ressemblent. Cela semble être le cas pour Yaniv, déjà engagé dans différentes luttes au moment où il échappe à l'armée et pour qui le fait d'être étiqueté « Profil 21 » constitue une étape logique dans un parcours déjà marqué par une certaine forme de marginalité.

Pendant les années qui suivent, Yaniv partage sa vie entre Israël et l'Europe. Il s'installe temporairement dans des squats de Berlin puis d'Amsterdam, où il fait l'expérience de la vie en communauté et se forme politiquement au contact de militants appartenant à la mouvance anarchiste $\mathrm{du}$ courant altermondialiste. Au début des années 2000, il participe à plusieurs mobilisations contre le G8, la Banque mondiale et le FMI, notamment à Prague et Gênes, où il est arrêté. On trouve dans le cas de Yaniv un bon exemple de la manière dont ces militants israéliens "multipositionnés » et souvent formés à l'étranger réimportent dans le champ de la lutte contre l'occupation des savoirs et des savoir-faire caractéristiques d'un capital militant transnational 54. En 2002, Yaniv s'engage au sein de l'ISM et il est envoyé à Naplouse protéger la maison d'un shabid, mot qui signifie « témoin » en arabe mais est généralement traduit par «martyr ». Dans le cas présent, il désigne quelqu’un qui a commis un attentat suicide en Israël et dont la maison est susceptible d'être démolie en représailles par l'armée israélienne. Ce passage à l'autre camp, qui représente probablement l'une des formes d'engagement les plus extrêmes qu'un Israélien puisse choisir, témoigne de la rupture déjà effectuée à l'époque par Yaniv avec sa société d'origine, mais aussi avec le milieu « pacifiste » israélien. En effet, le répertoire d'action disponible à l'époque - c'est-àdire avant le début de la coopération entre comités villageois en lutte contre le mur et anarchistes israéliens - pour protester contre l'occupation se résume principalement à des activités de solidarité que Yaniv juge «trop paternalistes ». Il évoque ainsi sans le nommer le groupe $T a$ 'ayush, qui organisait régulièrement des convois de denrées diverses dans les territoires occupés et se confrontait à l'armée lorsqu'elle tentait de l'empêcher d'accéder aux villages :

«La raison pour laquelle je suis allé avec l'ISM c'est qu'il n’y avait pas une organisation israélienne radicale dans laquelle je me sentais bien. Je voulais quelque chose de clairement sur le terrain et pour se

51. Les futurs combattants sont, eux, classés « Profil 97 », le plus haut de tous.

52. Au sens où l'utilise Erving Goffman. Goffman E., Stigmate. Les usages socianx des handicaps, Paris, Éditions de Minuit, 1975 [1 ère édition en anglais : 1963], p. 13.

53. Ibid., p. 33.

54. Ils se rapprochent en cela de ce que Sydney Tarrow nomme les « cosmopolites enracinés». Voir Tarrow S., The new transnational activism, Cambridge, Cambridge University Press., 2005 , p. 42. 
confronter vraiment à l'occupation; pas seulement “charity”... Il n’y avait pas encore les “Anarchistes contre le Mur”. Il y avait quelques Israéliens qui allaient dans les territoires mais chacun à sa façon, avec ses contacts, etc. Très individuellement. »

Cet engagement au sein de l'ISM amène Yaniv à dissimuler sa nationalité israélienne. Il dispose en effet d'un autre passeport, français, qui lui permet de passer le checkpoint de Hawarra, à l'entrée de Naplouse ${ }^{55}$, et surtout de ne mettre en danger ni la famille qui l'abrite sans connaître sa situation, ni luimême :

\begin{abstract}
«Les coordinateurs palestiniens savaient que j’étais Israélien mais ils m'avaient dit : “Il faut le cacher”. [Q : C'était dangereux pour toi ?] Oui. C’était avant que les journaux palestiniens ne parlent des “Anarchistes contre le Mur”. À Naplouse, les seuls Israéliens dont les gens entendaient parler, c'était des colons ou des soldats. Et moi j'avais rien à prouver à moi-même ou aux Palestiniens du genre : "Il y a des Israéliens qui sont pour la paix...” J'étais là pour combattre l'occupation. N'importe comment, n’importe où ».
\end{abstract}

À partir de 2004, Yaniv participe au campement de Mash'a, puis aux manifestations de Biddu, Buddrus et Bil'in. Il y fait l'expérience de la répression exercée par l'armée israélienne. À cette époque, il n'est pas rare que les routes d'accès soient bloquées plusieurs heures avant les manifestations. Les personnes extérieures doivent donc s'y prendre la veille pour arriver et dormir sur place. Régulièrement, les soldats envahissent les villages la nuit, entrent dans les maisons, arrêtent des gens suspectés, selon la version officielle de Tsabal, de faire partie des lanceurs de pierre et, parfois même, tirent à balles réelles. Yaniv raconte avec un certain détachement comment l'armée lui a tiré dessus en 2005 alors qu'il était dehors pendant un couvre-feu à Bil'in et ajoute qu'aujourd'hui « les manifs là-bas c'est gentil, c'est mignon, mais à l'époque, on s'ennuyait pas».

D’une manière plus générale, le rapport à la violence des Israéliens appartenant à cette catégorie de "militants multipositionnés » est souvent marqué par une sorte de mise à distance visant à dédramatiser les situations auxquelles ils se trouvent confrontés. La plupart n'hésite pas à s'exposer physiquement à des risques particulièrement élevés, arguant que cette exposition est non seulement contrainte par la brutalité de l'armée, mais justifiée par la nécessité de

55. Depuis les accords d’Oslo, les territoires occupés ont été divisés en trois zones. La zone A, qui comporte les villes palestiniennes, est théoriquement interdite d'accès aux Israéliens. Les Palestiniens citoyens d'Israël continuent néanmoins de s'y rendre, ainsi que certains militants israéliens juifs, notamment depuis le développement d'initiatives conjointes de lutte contre l'occupation. Les ressortissants étrangers ont, eux, la possibilité de se déplacer dans les différentes zones. 
se mettre à égalité avec les Palestiniens qui, eux, subissent quotidiennement l'oppression des forces occupantes. Certains vont même à la rencontre du danger, non pas, comme le sous-entendent parfois leurs détracteurs, en provoquant des soldats, mais plutôt en dédaignant d'employer les méthodes d'évitement de la violence choisies par d'autres : rester à distance des soldats, fuir lorsque les tirs commencent à pleuvoir, etc., sans même parler de négocier quoi que ce soit avec l'armée. L'engagement de ces militants peut, à plusieurs égards, être appréhendé comme une forme d'engagement total dont l'impact s'observe à tous les niveaux de la vie sociale des acteurs concernés et dont les coûts peuvent avoir des effets surrégénérateurs ${ }^{56}$. Vivre des situations risquées, accepter de se mettre en danger " pour la cause », sacrifier des plaisirs égoïstes au profit du bien collectif peut ainsi être vécu sur un mode substantiel comme apportant autant que cela coûte.

Mais, même si les coûts de la mobilisation peuvent être générateurs de plaisir, on peut se demander ce qui « fait tenir » ces « militants multipositionnés » et ce qui peut être à l'origine de leur désengagement. Pour la plupart âgés de 15 à 35 ans, ils habitent généralement à Tel-Aviv et bénéficient d'un fort potentiel de temps libre, ce qui peut permettre d'expliquer que, tout en étant élevés, les coûts - notamment temporels - liés à leur engagement ne soient pas insurmontables. Certains choisissent de professionnaliser leur engagement en travaillant par exemple pour des ONG ou des mouvements comptant des salariés, à la différence de Yaniv qui se montre réticent à devenir un « militant professionnel ». La plupart d'entre eux trouve des manières de combiner activités rémunérées et militantisme. Certains sont ainsi photographes et vendent leurs clichés aux médias ${ }^{57}$. D'autres travaillent pour des cabinets d'avocat défendant les militants inculpés, les villages en lutte contre la barrière, les organisations pacifistes inquiétées par le gouvernement, etc. L'interchangeabilité et la connivence des activités professionnelles et militantes permettent ainsi aux acteurs de s'attacher et de se détacher consécutivement de ces deux «fronts ». Yaniv, par exemple, a trouvé l'occasion d'investir professionnellement ses dispositions militantes en filmant les manifestations en tant que documentariste, position facilitée par sa détention d'une carte de journaliste.

L'ancienneté dans la carrière s'explique ainsi en partie par la capacité du « militant multipositionné » à investir des dispositions militantes dans le domaine professionnel. Le fait de se désengager, temporairement ou définitivement, de la participation aux manifestations contre la barrière peut, quant à lui, s'expliquer par le choix d'investir de manière plus intensive une autre des

56. Gaxie D., «Economie des partis et rétribution du militantisme », Revue Française de Science Politique, vol. $27, \mathrm{n}^{\circ} 1,1977$, p. 140.

57. C'est notamment le cas des membres du collectif de photographes militants Activestills («Clichés en action »), établi en 2005 pour documenter les différentes luttes sociales et politiques en Israël/Palestine. 
ramifications du militantisme. De nombreux Israéliens engagés à Bil'in se sont par exemple impliqués de manière très active dans la défense des demandeurs d'asile et réfugiés arrivés en Israël par le désert du Néguev à partir de 2007. Yaniv, pour sa part, a quitté le pays à la même époque. Il effectue régulièrement des séjours plus ou moins longs en Israël/Palestine et continue de participer, à ces occasions, à des actions conjointes. Son engagement principal se situe néanmoins aujourd'hui dans d'autres luttes qui, tout en convergeant avec les principes qui avaient guidé sa participation aux manifestations contre la barrière, rencontrent d'autres enjeux et l'amènent à côtoyer un autre milieu militant.

\section{"C'est ici qu'il faut être ». Le devoir d'engagement d'un militant pragmatiste}

Ido est né en 1981 et il est aujourd'hui étudiant de second cycle à l'Université de Tel-Aviv. Son histoire familiale apparaît d'emblée comme un élément explicatif de son engagement contre la barrière de séparation. Dans les années 1970, ses parents ont été membres du Matzpen, un groupe israélien trotskyste anti-sioniste et, en 1988, son père a refusé de servir pendant la première Intifada. Pourtant, quand le moment est arrivé pour lui de rejoindre l'armée en 1998, Ido s'est enrôlé dans une unité combattante, comme l'avaient fait ses frères avant lui. Le climat politique dans lequel a grandi Ido, qui avait 12 ans lorsque les accords d'Oslo ont été signés, et la situation relativement calme de la période à laquelle il entre dans l'armée permettent en partie d'expliquer ce parcours militaire en décalage avec une socialisation familiale d'extrême-gauche, comme il le souligne lui-même :

«Mes parents ne sont pas sionistes. Quand ils étaient jeunes, ils étaient comme les “Anarchistes contre le Mur”, même plus radicaux pour l'époque. Ils étaient des trotskystes purs et durs. C'est très bizarre que mes frères et moi soyons devenus des soldats combattants. C'est même complètement fou. [...] Quand j'ai été appelé, j'avais 18 ans. Je savais que mon père était allé en prison en 1988 pour avoir refusé de servir dans les territoires. Donc j'avais entendu parler de l'objection de conscience... Mais l'unité dans laquelle j'étais n'allait pas dans les territoires occupés à ce moment-là et la situation, en 1998, n’avait rien à voir avec celle de maintenant. »

Son service militaire terminé, Ido part plusieurs mois en Asie ${ }^{58}$ avant de revenir en Israël et commencer ses études. C'est alors que le groupe «Le Courage de Refuser » (HaOmetz Lesarev) fait paraître une lettre dans un grand quotidien israélien, dans laquelle une cinquantaine d'officiers appellent au refus de servir dans les territoires occupés. Ido explique ce qui l'a poussé à signer cette lettre :

58. Il est très fréquent pour les jeunes Israéliens de partir plusieurs mois en Asie, en Amérique du Sud ou en Océanie une fois leur service militaire terminé. 
"Après être revenu d'Extrême-Orient, "Le Courage de Refuser" commençait juste ses actions. C’était en janvier 2002. J'ai lu ce qu'ils avaient écrit. J'étais plus conscient et [...] je me suis reconnu dans leur message même si, dans "Le Courage de Refuser", il y a tout le côté sioniste. Je n'étais pas vraiment là-dedans mais je me suis dit que c'était important d'en faire partie [...] pour augmenter le nombre de signatures. »

Comme il le souligne lui-même, le groupe auquel il adhère alors se distingue par sa mobilisation des schèmes sionistes : le slogan principal du «Courage de Refuser » est « refuser, c'est sioniste » et son logo représente une étoile de David aux couleurs du drapeau d'Israël. Bien que tous ses signataires ne se retrouvent pas, loin s'en faut, dans ce patriotisme affiché, se positionner ainsi, tout comme rappeler leurs faits d'arme glorieux devant les médias, relevait à l'époque d'une volonté des initiateurs de la lettre de ne pas apparaître comme des lâches ou des traîtres. Pour une personne comme Ido ayant grandi dans une famille anti-sioniste - un positionnement extrêmement rare chez les Israéliens de descendance juive -, adhérer à un groupe sioniste représente une nouvelle prise de distance vis-à-vis de l'engagement passé de ses parents, qu'il ne renie pas pour autant.

A la même période, il participe à quelques activités du groupe Ta'ayush et se porte volontaire dans l'ONG B'tselem. En 2005, une amie de l'université lui propose de l'accompagner à Bil'in. Pendant un an, il se rend de temps en temps dans le village, où il prend part aux manifestations contre la barrière avant de s'engager pleinement au sein des «Anarchistes contre le Mur » et dans la lutte conjointe avec les Palestiniens. Il explique les raisons qui l'ont amené non seulement à devenir un « habitué » de Bil'in mais aussi à participer aux réunions et autres activités du groupe :

«Au début, je venais une fois de temps en temps mais après un certain temps, quand tu connais les gens, tu te dis : "Si je suis dedans, alors je dois être dedans à fond !” Maintenant, j’y consacre tout mon temps libre sauf quand je suis coincé à cause d'un travail pour l'université... Ça prend beaucoup de temps d'aller là-bas constamment, chaque semaine et puis, ce n'est pas seulement le vendredi ; ce sont aussi les réunions et, quand tu as des responsabilités, c'est 24/24h. Tu ne peux pas être à moitié dedans... Je veux dire qu'en ce qui me concerne, je ne peux pas venir seulement une fois par semaine à Bil'in et c'est tout, comme je le faisais au début. Si je voulais être moins actif, il faudrait que je sois vraiment très loin, parce que je me sens engagé envers les gens, les Palestiniens mais aussi le groupe. »

Ici, ce sont les contacts créés avec les autres militants, Israéliens et Palestiniens, qui viennent renforcer un engagement initialement quelque peu 
détaché et faire d'Ido un manifestant contre la barrière « à part entière ». Pour autant, il se distingue fortement des "militants multipositionnés » comme Yaniv. En effet, bien qu'il ait été socialisé à l'extrême-gauche de l'échiquier politique israélien, il se sent différent des membres à l'origine du groupe, ceux qui ont justifié son appellation en «Anarchistes contre le Mur » :

« Je ne suis pas un anarchiste, ce n'est pas mon genre de truc, ça se voit directement. Je suis bien plus proche des communistes. J'apprécie beaucoup les membres de ce groupe et ce qu'ils font, mais dans n'importe quelle autre situation, jamais je n'aurais fait partie d'un groupe anarchiste. Je n'étais pas très engagé dans Ta'ayush à l'époque mais c'est un groupe qui me ressemblait plus.»

Suite au déclenchement de la seconde Intifada à l'automne 2000, la situation humanitaire était devenue catastrophique. De nombreux villages avaient été placés sous couvre-feu, subissaient des bouclages à répétition, étaient privés des denrées les plus élémentaires et faisaient l'objet d'incursions incessantes de Tsabal. C'est dans cette situation d'urgence que le groupe Ta'ayush avait été créé par des Juifs et Arabes israéliens pour mener des actions de protestation et de solidarité avec les Palestiniens des territoires 59 . Après plusieurs années d'activité, $T a$ 'ayush a cessé la plupart de ses activités. Un petit noyau de membres actifs continue de se rendre dans la région des collines du sud d'Hébron pour aider les Palestiniens, mais ces militants admettent que ce qui subsiste du groupe aujourd'hui n'a plus rien de commun avec ce qu'il était pendant les premières années de l'Intifada.

Le délitement de Ta'ayush et, avec lui, la disparition de l'une des seules possibilités d'actions directes non-violentes et conjointes a laissé un certain nombre de militants israéliens « orphelins 60 ». Ido ne faisait pas partie de ceux qui étaient fortement impliqués dans ce groupe ; il ne participait que ponctuellement à ses activités. Néanmoins, il souligne lui-même que Ta'ayush lui « ressemblait plus » car son répertoire d'action mêlant aspect humanitaire et dimension politique permettait d'agir concrètement pour aider les Palestiniens tout en s'opposant fermement à l'armée. L'entrée d'Ido dans une carrière militante à haut risque à travers la participation aux manifestations contre la barrière semble donc plus s'expliquer par la convergence d'un « besoin de faire quelque chose de concret » et d'une absence d'alternative satisfaisante dans le champ militant anti-occupation à un moment donné que par une identification forte avec les «Anarchistes contre le Mur ». D'ailleurs, Ido se verrait bien dans le futur "faire quelque chose comme les "Anarchistes" mais avec des gens qui me ressemblent plus et sont moins orientés anarchistes, des gens du

59. Pour plus d'informations sur Ta'ayush, voir Schulman D., Ta'ayush : journal d'un combat pour la paix Israël-Palestine, 2002-2005, Paris, Seuil, 2006.

60. Plusieurs personnes ont, au cours d'entretiens ou de discussions informelles, utilisé ce mot pour qualifier ce qu'ils ont ressenti suite à la disparition de Ta'ayush. 
Chadash 61 ou de l'université par exemple ». L'apparition de nouvelles offres sur le marché militant lui correspondant plus semble donc être l'une des voies par lesquelles un « militant pragmatiste » comme Ido peut être amené à se désengager.

L'expérience de la violence peut également jouer en faveur d'une désaffection plus ou moins progressive des militants qui y sont confrontés régulièrement. Dans le cas d'Ido, elle semble au cœur d'un processus qui l'amène à apprivoiser non seulement sa peur, mais aussi sa colère à l'égard des soldats, très vive au début et aujourd'hui plus distanciée. Quelques jours avant notre second entretien, son frère avait été assez sérieusement blessé à Bil'in. Ido m'expliquait cependant qu'il ne ressentait plus, comme au début, un désir de vengeance à l'égard des soldats :

« Il y avait une période où, quand les soldats étaient très violents à Bil'in, j'étais vraiment en colère contre eux et ça me donnait envie d'aller lancer des pierres avec les shababs ${ }^{62}$, de frapper, etc. Ils étaient complètement fous, sauvages et moi, ça me filait la rage... Mais aujourd'hui, $j$ 'ai changé. Il y a un mois, j’ai été frappé très violemment à l'aide d'un bâton par un soldat. Ca m'a fait vraiment mal mais je n'ai pas ressenti de colère. Et même après qu'on a tiré sur mon frère, je n'ai pas eu cette rage que j'avais au début. »

Dans le cas présent, la capacité à ne pas tomber dans le piège de l'engrenage fonctionne comme une manière de réaffirmer la légitimité d'un engagement socialement dévalué autant que l'iniquité de la situation. Pour Ido comme pour d'autres, tout d'abord socialisés au militantisme anti-occupation à travers des actions moins susceptibles de dégénérer que les manifestations contre la barrière, Bil'in et les autres villages sont des lieux où des valeurs telles que la non-violence se heurtent à la réalité concrète du terrain. Certains s'y découvrent une haine des soldats alors même qu'ils mettaient jusqu'alors un point d'honneur à distinguer les décideurs politiques de ceux qui appliquent les ordres, tandis que d'autres, convaincus par la cause avant même de venir, s'en désolidarisent lorsqu'ils voient de jeunes Palestiniens lance-pierre à la main.

\section{"Je veux garder ma naïveté ». L'engagement improbable d'un militant néo- phyte}

Une trajectoire comme celle de Doron est relativement rare parmi les militants contre la barrière. Si elle n'est pas marquée directement par une rupture avec la socialisation familiale, dans le sens d'un passage de la droite à la gauche,

61. Parti communiste israélien.

62. «Jeunes» en arabe. 
elle se caractérise par plusieurs éléments qui le distinguent de la majorité des autres militants. Doron est né en 1986 dans une petite ville de la banlieue résidentielle de Tel-Aviv. Ses quatre grands-parents avaient immigré d'Irak en Israël dans les années 1950 et vécu dans des camps de transit avant de s'installer dans des logements en dur. Son père, décédé alors que Doron avait sept ans, était artisan et sa mère est toujours secrétaire médicale. Il décrit son éducation comme «complètement apolitique » et explique que, dans sa famille, le sionisme se manifeste plus par une sorte d' "amour naif du pays » que par un nationalisme exacerbé. De même le rapport à l'armée y est plus utilitaire qu'idéologique :

\begin{abstract}
«Personne ne m'a appris que je devais aller à l'armée et mourir pour mon pays, ou être combattant. Dans ma famille, ce n'était pas important d'aller à l'armée pour servir le pays mais parce que ça fait partie du cours normal de la vie en Israël et que si tu ne vas pas à l'armée, tu risques d'être considéré comme bizarre, de ne pas trouver de travail, etc. [...] Pour moi, aller à l'armée n'avait rien d'une chose naturelle, contrairement à beaucoup de jeunes. Je m'en fichais complètement et je ne pensais pas que c'était une bonne chose ni même une chose nécessaire. »
\end{abstract}

Le fait que Doron ait grandi dans une famille irakienne peut contribuer à expliquer en partie la distance de ses parents vis-à-vis de l'institution militaire, elle-même constitutive de son propre désintérêt pour la question. En Israël, en effet, l'armée est restée pendant des décennies le bastion des descendants des pionniers ashkénazes, laïcs et socialistes qui avaient dirigé la communauté juive de Palestine avant 1948, puis occupé les postes à responsabilité dans le nouvel État. Aujourd'hui encore, les soldats servant dans les unités les plus prestigieuses de l'armée sont, dans leur grande majorité, des Achusalim 63. Cet acronyme, formé en hébreu par les mots "ashkénazité" (ashkenaziut), laïcité (chiloniut), socialisme (sozialism) et nationalisme (leumanut) désigne les caractéristiques principales de l'élite politico-militaire israélienne qui a dirigé le pays jusque dans les années $1980{ }^{64}$. On peut supposer que les parents de Doron, socialisés en Israël mais dont les parents étaient eux-mêmes arrivés d'Irak dans les années 1950, n'ont pas développé le même attachement à l'armée que les descendants de Sabras 65 bercés toute leur enfance par des récits de combats, de fraternité entre soldats et de sacrifice pour le pays. De plus, même si son père a participé aux guerres de 1967 et 1973, Doron l'a perdu trop tôt pour avoir l'occasion de l'entendre évoquer ce sujet. Enfin, étant le cadet de trois sœurs, il n'a pas grandi dans un environnement préoccupé du devenir militaire des jeunes de la famille.

63. Kimmerling B., Ketz shilton haAbusalim [La fin de l'hégémonie ashkénaze], Jérusalem, Keter, 2001.

64. Décennie à partir de laquelle des Juifs orientaux et des Juifs religieux ont commencé à occuper des postes à responsabilité dans l'armée et au gouvernement. 
Lorsque la seconde Intifada éclate, Doron a 14 ans et chaque après-midi, en rentrant de l'école, il allume la télévision pour voir combien de personnes ont été tuées par des attentats. Pour autant, il ne développe pas, à cette époque, d'animosité particulière à l'égard des Palestiniens et ce alors même que les discours témoignant d'une peur des «Arabes 66 » étaient fréquents dans sa famille. Vers l'âge de 16 ans, il commence à lire les articles de Yediot Acharonot traitant du conflit israélo-palestinien. Bien que ce quotidien ne soit pas connu pour des prises de position particulièrement progressistes, Doron dit s'être formé politiquement à sa lecture car il y découvre notamment les écrits de B. Michael et de Meir Shalev, deux journalistes de gauche. Pour autant, ses opinions politiques ne sont pas encore très claires à l'époque; il parle d'ailleurs de mishmash (" méli-mélo ») pour les qualifier. Plusieurs années de suite, il participe à des camps de vacances en Europe, où il rencontre des jeunes d'un peu partout et prend conscience que l'importance accordée à l’armée dans le parcours de vie est très israélienne.

Quand arrive le moment de l'enrôlement, il fait son possible pour être exempté sans se voir attribuer le fameux « Profil 21 ». Doron, qui possède déjà un bon dossier scolaire, veut en effet faire des études scientifiques poussées et craint qu'une objection de conscience dissimulée sous des motifs psychologiques ne diminue fortement ses chances d'atteindre cet objectif. Il finit donc par servir en tant que soldat non-combattant dans différentes bases militaires situées à proximité de chez lui et, son service obligatoire terminé, il s'inscrit à l'université. Quelques mois plus tard, il reçoit un courriel du syndicat étudiant lui proposant de participer à un « tour 67 » des barrages militaires organisé par Machsom Watch ${ }^{68}$ en Cisjordanie. Il saisit l'occasion :

«J'ai pensé que c'était une bonne opportunité. Je voulais voir la Cisjordanie, j'aimais bien les tours, c'était gratuit alors je me suis inscrit sur la liste et j'y suis allé. C'était un vendredi. [...] À la fin du tour, on s'est arrêté dans un café à Hawarra, où on a rencontré trois étudiantes palestiniennes de l'université américaine de Jénine qui étaient là avec leurs professeurs. On a parlé avec elles pendant plus d'une heure et pour moi, ça a été un moment très important. C'était la première fois que je rencontrais des Palestiniens avec qui je pouvais avoir une interaction personnelle et une discussion politique. »

65. Ce nom, dérivé du terme hébreu Tsabar («figue de barbarie ») désigne les Juifs nés en Palestine avant 1948 et plus tard en Israël.

66. Ses grands-parents avaient notamment connu des pogroms en Irak et en gardaient une forte méfiance à l'égard des «Arabes » d'où qu'ils soient.

67. Siour en hébreu. Ces visites de lieux emblématiques de l'occupation (mur de séparation, checkpoints, colonies, ville de Hébron, etc.) sont organisées par certaines organisations israéliennes pour informer les Israéliens ainsi que les visiteurs étrangers de la situation dans les territoires occupés.

68. Combinaison du mot hébreu machsom («barrage militaire ») et du mot anglais watch (« observatoire ). Machsom Watch est une organisation israélienne formée en 2002 par des 
Au-delà de l'aspect militant, cette première expérience plaît aussi à Doron car elle lui permet de rencontrer des personnes dont il se sent plus proche que celles auxquelles il a généralement accès par ses autres réseaux de sociabilité, notamment des filles ${ }^{69}$. Quelques semaines après sa première expérience militante, Doron participe à un autre «tour ", organisé cette fois par l'organisation $\operatorname{Ir~} \mathrm{Amim}^{70}$ puis, à l'automne 2009, il se rend à quatre reprises dans les territoires palestiniens pour cueillir des olives ${ }^{71}$. Sa description de cette expérience montre qu'il a bien conscience de la manière dont les bénéfices secondaires ont pu jouer dans sa motivation de poursuivre son engagement :

\begin{abstract}
« Je trouvais ça sympa et intéressant. Ça me permettait de rencontrer des Palestiniens avec qui je pouvais discuter. Je voulais connâ̂tre des gens et avoir de nouvelles expériences. Et jusqu'à aujourd'hui, chaque fois que je vais dans une action, il m'arrive quelque chose de stimulant : je rencontre quelqu'un d'intéressant, j'apprends quelque chose que je ne savais pas, je mange quelque chose de nouveau, je fais quelque chose de particulier... Bref, à côté de toutes les contraintes, il $\mathrm{y}$ a des choses très attrayantes pour moi dans le fait d'être militant. »
\end{abstract}

En octobre 2009, il se rend à un «tour » de la ville d'Hébron. C'est à cette occasion que je fais sa connaissance. Suite à une longue discussion où je lui explique sur quoi porte mon travail de thèse et où il m'expose sa courte trajectoire militante - il n'est alors engagé que depuis six mois - il me demande si je peux le prévenir la prochaine fois que je me rends dans une manifestation contre la barrière. C'est ainsi à mes côtés qu'il découvre le village de Bil'in et ses habitants.

« J'avais entendu parler des manifs à Bil'in, au moment de l'affaire Gil Naamati, mais je pensais juste que c'était quelque chose de trop extrême, de dangereux et qui ne me correspondait pas [...] Plus tard,

femmes qui surveillent le comportement des soldats aux barrages militaires de Cisjordanie et de Jérusalem Est.

69. Les sociologues des mobilisations ont accordé une attention particulière aux liens sociaux comme facilitateurs de l'engagement, la connaissance antérieure de personnes engagées expliquant en partie la participation aux actions collectives (voir McAdam D., op. cit. ; Paulsen R., "Specifying the Relationship between Social Ties and Activism", American Journal of Sociology, 99, 3, 1993, pp. 640-667). Le développement de nouvelles sociabilités et liens affectifs dans le cercle militant contribue aussi souvent au maintien de l'engagement, même s'il est également susceptible de contribuer au désengagement (Goodwin J., "The Libidinal Constitution of a High-Risk Social Movement: Affectual Ties and Solidarity in the Huk Rebellion”, American Sociological Review, 62, 1, 1997, pp. 53-69).

70. «La cité des peuples». Organisation israélienne fondée en 2004 qui se concentre sur les problèmes spécifiques à Jérusalem et cherche notamment à promouvoir plus d'égalité dans cette ville.

71. Octobre et novembre sont les mois de cueillette des olives. Depuis plusieurs années, des groupes israéliens comme les «Rabbins pour les Droits de l'Homme » (Rabanim lema'an Schuyot Adam) ou la «Coalition pour la Cueillette des Olives » (Coalitzia haMassik) organisent le transport en autocar de volontaires qui viennent aider les villageois palestiniens. 
quand j'ai commencé à devenir militant, j'y ai pensé mais... Le mot “anarchiste" est un mot qui fait peur pour quelqu'un qui n'est pas de ce milieu. Et puis, j'avais entendu parler de la répression, des gaz lacrymogènes, des balles en caoutchouc... Je ne savais pas si c'était une bonne idée. Mais quand je t'ai rencontrée, tu m'as dit que tu y étais allée souvent mais que tu avais toujours peur. C'était parfait pour moi parce qu'à la fois, tu savais où tu allais et tu cherchais à être en sécurité. Donc je me suis dit que si je voulais y aller, c'était avec toi ou jamais!»

Cette situation dans laquelle, tout en enquêtant sur l'engagement israélien contre l'occupation, je suis devenue partie prenante du processus étudié, a bien entendu été l'objet de nombreux questionnements méthodologiques mais elle a surtout été particulièrement riche, du fait même de son caractère liminal. Grâce à l'amitié nouée avec ce « militant néophyte », j'ai pu suivre un parcours d'entrée en militance depuis son commencement, ainsi que les changements de perspective et les questionnements qui l'ont accompagné. Au cours de sa troisième visite à Bil'in, Doron fait la rencontre d'un habitant du village, Issa, qui l'invite chez lui à plusieurs reprises et avec qui il a d'interminables discussions sur la politique, la vie sous occupation, la culture palestinienne, etc. Bien qu'ils aient une dizaine d'années d'écart et des situations familiales très peu semblables - Doron est célibataire et vit toujours chez sa mère, tandis qu'Issa est marié et père de quatre enfants - une complicité se développe rapidement entre les deux jeunes hommes 72 et contribue à fidéliser l'engagement du premier dans le village du second:

«Aujourd'hui, je pense que si je continue de venir à Bil'in, c'est en partie à cause de ma familiarité et de mes interactions personnelles avec des gens là-bas. Peut-être que si je n'avais pas rencontré [Issa], j'aurais arrêté d'y aller au bout d'un moment. »

Dans le cas de Doron, il est intéressant de noter que les liens tissés à travers l'engagement le sont avec des Palestiniens et très peu avec d'autres Israéliens engagés. Cela semble s'expliquer autant par une fascination liée à la découverte d'un monde jusque-là étranger, que par une impression d'étrangeté ressentie à l'égard des autres militants :

« Je n'ai pas l'impression d'être comme ces anarchistes dont parlent les médias qui viennent, foutent le bazar et s'en vont. Bien sûr, c'est une image fausse mais je me sens différent. Déjà je ne vis pas à TelAviv. Et puis, dans la communauté anarchiste, les questions queer et anti-spécistes sont très importantes et personnellement, ça ne me

72. Issa parle parfaitement hébreu car il a travaillé plusieurs années en Israël avant la seconde Intifada. C'est donc dans cette langue qu'ils communiquent, bien que Doron possède quelques rudiments d'arabe grâce à ses grands-parents. 
touche pas. Je ne suis pas anarchiste. Je me sens solidaire de certaines idées anarchistes mais ce n'est pas pour ça que je me bats. Je me bats pour les droits de l'homme [...]. Et puis, je veux garder ma naïveté. Je ne veux pas protester pour protester. Je veux rester un Israélien qui proteste quand c'est nécessaire. »

En mai 2010, Doron est arrêté pour la première fois lors d'une manifestation dans les environs de Jérusalem et inculpé pour avoir insulté les soldats, alors qu'il tentait de discuter avec eux. Cet épisode est particulièrement mal vécu par le jeune homme qui cesse pendant quelques semaines d'aller dans les territoires occupés. Les pressions familiales pour qu'il abandonne le militantisme, ainsi que le risque de ne pouvoir accéder aux formations universitaires convoitées en raison de son casier judiciaire ne le convainquent pourtant pas car, dit-il, « devenir militant était la chose la plus naturelle pour moi ; j'ai toujours su, au fond de moi, que j'étais de gauche ». L'entrée dans une carrière de militant contre l'occupation peut donc en quelque sorte s'appréhender, chez Doron, comme une « sortie du placard » qui ne laisse que peu de retour possible en arrière. La participation plus spécifique aux manifestations contre la barrière pourrait, elle, être remplacée par des formes moins risquées de militantisme si les liens créés à Bil'in ne rendaient pas le désengagement affectivement et moralement si coûteux.

$$
\text { *** }
$$

A travers la présentation de trois carrières de manifestants israéliens contre la barrière de séparation, cet article a tenté d'illustrer quelques-uns des chemins menant à cet engagement militant. Pour autant, il ne prétend nullement avoir épuisé la diversité des cas, ni leurs spécificités. Plus encore que la catégorie de «militant », celle de « manifestant » est labile et poreuse : certains viennent une fois et ne reviennent plus; d'autres participent seulement de temps en temps aux protestations contre la barrière, s'investissant par ailleurs dans d'autres formes de contestation; quelques-uns encore sont « fidèles au poste » depuis plusieurs années. Les trois militants ici présentés ont en commun d'avoir développé des liens importants avec les villageois palestiniens en lutte et/ou avec d'autres militants israéliens et d'avoir ainsi fait d'une participation aux manifestations qui aurait pu ne rester que ponctuelle un engagement continu dont les conséquences se ressentent à différents niveaux de leur vie sociale.

Les risques et les coûts accompagnant cet engagement sont élevés, ce qui explique que les analyses en termes de rétributions ne suffisent pas à comprendre ce qui amène des Israéliens à participer à ces manifestations. Le modèle proposé par Doug McAdam sur le recrutement dans le militantisme risqué et coûteux permet de montrer ici comment dispositions idéologiques, contacts 
préalables avec des militants et disponibilité biographique s'articulent dans ces trois types de trajectoires.

Si la transmission, au sein de la famille, de valeurs et d'attitudes politiques constitue sans aucun doute l'un des éléments explicatifs de l'engagement, d'autres types d'apprentissages sont « constitutifs d'un rapport politique au monde social » ${ }^{73}$. Ainsi, rien ne « prédisposait » Doron à devenir un opposant actif à la barrière de séparation, si ce n'est une sensibilité à la question des droits de l'homme ainsi qu'un esprit scientifique qui, dit-il, ne lui permet pas de «comprendre comment on peut rationnellement soutenir l'occupation ». Dans les deux autres cas, on est certes face à des militants socialisés politiquement de manière précoce, mais voir dans leur engagement l'aboutissement mécanique de leur politisation reviendrait à conclure que les mêmes causes entrainent les mêmes effets. Analyser les carrières préalables à l'engagement contre la barrière avait ainsi pour but d'éviter cet écueil.

Par ailleurs, pour comprendre ce qui guide les acteurs sociaux vers telle ou telle forme de militantisme, ce qui les amène à s'identifier plus ou moins à tel ou tel groupe militant et à préférer certains répertoires d'action à d'autres, la prise en compte de l'offre militante disponible à un moment donné semble déterminante, de même que celle du facteur temps. Une grande partie des militants contre la barrière engagés dès 2004 partagent, à l'instar de Yaniv, un ensemble de caractéristiques contribuant à les différencier des militants arrivés par la suite, pendant la période 2005-2007 - comme Ido - et plus encore 2008-2010 - comme Doron.

Pour autant, il semble délicat de parler dans notre cas de «générations militantes » comme le fait par exemple Cécile Péchu ${ }^{74}$, dans la mesure où les manifestations contre la barrière n'existent que depuis six ans et où les militants partagent dans l'ensemble une certaine homogénéité générationnelle. Tout au plus peut-on souligner que les modalités différenciées prises par cet engagement dans les carrières étudiées s'expliquent en partie par la temporalité propre à chacune d'entre elles. Manifester aujourd'hui contre la barrière alors que les protestations ont lieu depuis plusieurs années, font l'objet d'un fort soutien international et ont été accompagnées, dans certains cas, de victoires juridiques n'a plus grand-chose à voir avec ce que cela représentait en 2004.

Le succès relatif de ces manifestations se mesure autant à leur régularité et à leur longévité qu'au symbole qu'elles sont parvenues à véhiculer. Si dans les années quatre-vingt et quatre-vingt-dix, les organisations du mouvement «pacifiste » parvenaient à attirer dans leurs rangs des dizaines de milliers de

73. Bargel L, «Socialisation politique ", in Fillieule O., Mathieu L., Péchu C., (eds.), Dictionnaire des mouvements sociaux, Paris, Presses de Science Po, 2010, p. 511.

74. Péchu C., op. cit. 
manifestants réguliers et à délivrer un message ayant la capacité de résonner auprès d'un grand nombre d'Israéliens, les mobilisations qui ont accompagné et suivi la seconde Intifada témoignent d'une nouvelle manière d'envisager la lutte et les rapports entre Israéliens et Palestiniens. De Bil'in à Hébron, la coopération se veut aujourd'hui horizontale mais aussi transgressive : non seulement les militants des deux côtés de la « ligne verte» sont devenus des «partenaires de lutte », mais ils unissent leur force face aux soldats envoyés par le gouvernement israélien pour réprimer les protestataires. Ce faisant, ils tracent un chemin vers la reconnaissance mutuelle des deux peuples qui ne se contente plus d'être un principe politique mais qui influe sur leurs manières d'agir et de penser. Les passages opérés par Yaniv, Ido, Doron et les autres militants d'un côté à l'autre de ce mur/barrière érigé par Israël en frontière (physique, mais aussi et surtout mentale) et leurs rencontres avec des Palestiniens contribuent d'ailleurs à expliquer qu'ils soient prêts à payer cet engagement de leur intégrité physique et mentale. 